\title{
Large-Area Phase Mapping Using PhAse Recognition and Characterization (PARC) Software
}

\author{
Corrie van Hoek, ${ }^{*}$ James Small, and Sieger van der Laan
}

Tata Steel, R\&D, Microstructure \& Surface Characterization (MSC), P.O. Box 10.000, 1970 CA IJmuiden, The Netherlands

*corrie.van-hoek@tatasteel.com

\begin{abstract}
The spectral imaging (SI) approach is used in analytical electron microscopy for determining chemical compositions in materials at the microscale. A major challenge is how to efficiently unlock microspatially resolved chemical information in large SI data cubes. Tata Steel has developed an in-house software approach called $P h A s e$ Analysis, Recognition and Characterization (PARC). PARC combines automated phase recognition with flexible user-defined refinement functions to create phase allocation models. These models may be used in automated batch processing on multiple SI fields, enabling the visualization of complex microstructures, and the quantification of phase proportions and chemistry, at length scales up to several millimeters. The approach bridges the gap between microanalysis and bulk analysis and lends itself to cross-validation with independent bulk analytical techniques such as X-ray fluorescence (XRF) and X-ray diffraction (XRD).
\end{abstract}

\section{Introduction}

Scanning electron microscopy (SEM) combined with $\mathrm{X}$-ray microanalysis is widely used for the characterization of solid materials. With the current generation of hardwareincluding field-emission electron guns, energy-dispersive X-ray analysis systems employing silicon drift detectors (SDD), and fast acquisition capabilities - chemical information from each pixel in an SEM image can be acquired within milliseconds. The greatest challenge is how to efficiently extract the maximum information from such a spectral imaging (SI) data set. Locked within the acquired SI data is spatially resolved chemical information that can be used to define phases, map their distribution and relative abundances, and determine the accurate chemistry of not only the individual phases but also the local bulk composition on the millimeter to centimeter scale. To unlock this potential at Tata Steel, we have developed the $\mathrm{Ph} A$ se Recognition and Characterization (PARC) postprocessing software for SI data [1-3].

The ability to determine the distributions and chemical compositions of individual phases is crucial for the characterization of materials. Phases, as opposed to elements, are the most directly relevant building blocks of many complex materials and are indicative of the conditions of a material's formation and its chemical and mechanical properties. The spatial distribution of phases can be related to gradients in chemistry or physical conditions like temperature and oxygen activity $\left(\mathrm{fO}_{2}\right)$. Often gradients in phase distribution become apparent only at a scale of centimeters (for example, in geological materials) requiring characterization of microstructure and microchemistry on this length scale. Moreover, phases can have internal chemical zonation. For tackling such materials characterization, the acquisition of SIs from large areas is particularly powerful, avoiding many of the pitfalls of manual point analysis and associated user bias.

To illustrate the application of PARC, we use it to study the microstructure of metallurgical slag produced in steelmaking. The studied slag derives from the converter process in which liquid $\mathrm{Fe}$ metal from the blast furnace is refined by extracting
$\mathrm{C}, \mathrm{Si}, \mathrm{P}, \mathrm{Mn}$, and $\mathrm{Ti}$ as well as trace metals like $\mathrm{V}, \mathrm{Ba}, \mathrm{Cr}$, and Mo. In the converter, $\mathrm{C}$ forms gaseous $\mathrm{CO}$, and the other impurity elements are oxidized and react with lime $(\mathrm{CaO})$ and dolime $(\mathrm{CaO} . \mathrm{MgO})$ to form a slag. Converter slag is subject to European Union regulations regarding Registration, Evaluation, Authorisation and Restriction of Chemicals (REACH) [4]; it is the most voluminous byproduct of steelmaking $(\sim 0.1$ ton slag per 1 ton steel) with limited options for reuse. Understanding slag microstructure and phase makeup is relevant for predicting its environmentally relevant behavior [5]. Converter slag is particularly challenging to study because, owing to rapid nonequilibrium cooling, phase distribution gradients as well as chemical zonation within phases are present.

This article provides a description of the PARC software and its application to the characterization of converter slag. A more extensive comparison of PARC results with complementary methods of analysis such as X-ray diffraction-Rietveld (XRD-Rietveld) and X-ray fluorescence (XRF) bulk chemical analysis is the subject of a separate publication [6].

\section{Materials and Methods}

SI-data processing and the rationale for PARC. Each pixel within an SI data set stores a full energy dispersive $\mathrm{X}$-ray spectroscopy (EDS) spectrum, representing elemental information derived from $\mathrm{X}$ rays emitted from the electron beam interaction volume. Various options are integrated into the majority of commercial EDS-software packages



Figure 1: Reflected light microscopy image of K3-slag grains showing two grains selected for analysis with PARC. 


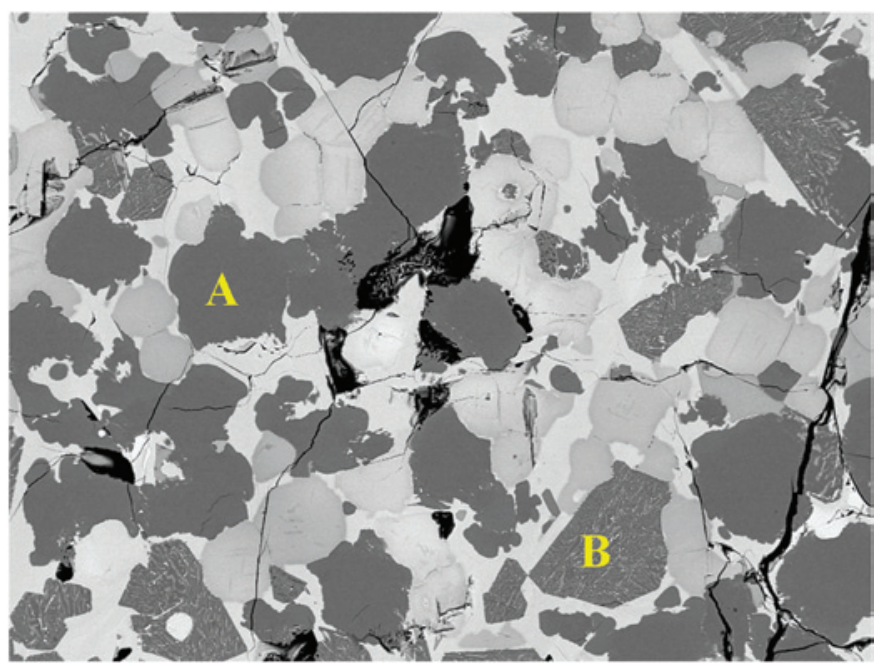

Figure 2: $\mathrm{BSE}$ image from a $\mathrm{K} 3$ slag. $\mathrm{A}=\mathrm{Ca}_{2} \mathrm{SiO}_{4}(\mathrm{C} 2 \mathrm{~S}), \mathrm{B}=$ decomposed $\mathrm{Ca}_{3} \mathrm{SiO}_{5}$ (C3S): phase intergrowth - dark phase $\mathrm{C} 2 \mathrm{~S}$, bright phase lime $(\mathrm{CaO})$. Image width $=512 \mu \mathrm{m}$.

to process, visualize, and utilize this spatially resolved information. Summed spectra from multiple pixels can be extracted using user-defined square-, circular-, polygon-, or BSE image-based selections. To visualize spatial variations in local chemical composition, X-ray maps of different user-selected elements can be extracted and reported in terms of net counts or weight (and atomic) percentages. These element maps can be overlaid on the reference SEM grayscale image. Because there are only three primary colors, the
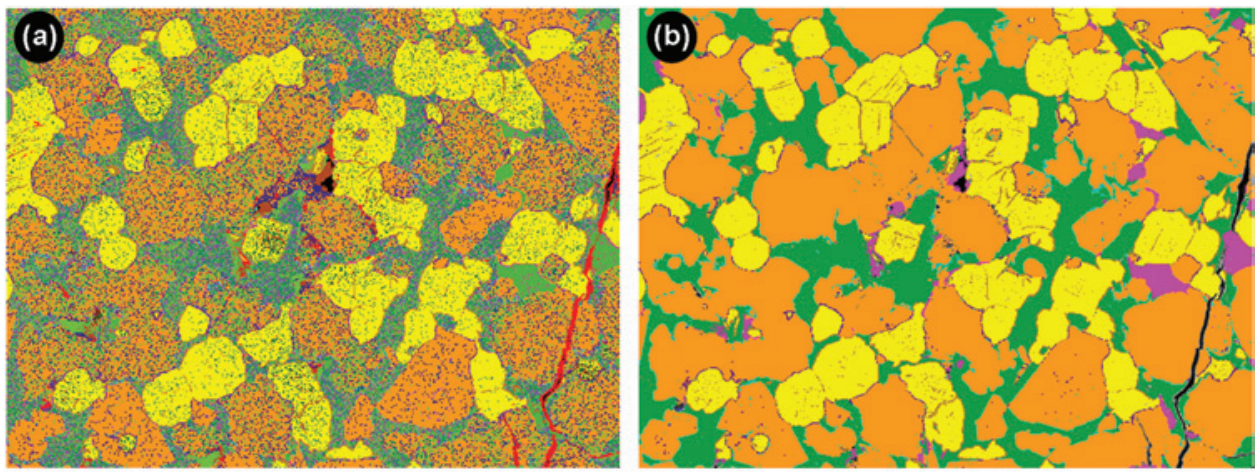

\begin{tabular}{|l|l|}
\hline (c) $)$ & Unclassified \\
& Empty Spectra \\
\hline & Embedding \\
\hline & CaSilicate \\
\hline & Mg-Wue $(\mathrm{Mg}, \mathrm{Fe}, \mathrm{Mn}) \mathrm{O}$ \\
\hline & Srebrodolskite \\
& $2 \mathrm{CaO}(\mathrm{Fe}, \mathrm{Cr}, \mathrm{Al}, \mathrm{V})_{2} \mathrm{O}_{3}$ \\
\hline & $\mathrm{CW} 3 \mathrm{~F}\left(\mathrm{CaO} .3 \mathrm{FeO}_{\mathrm{Fe}} \mathrm{O}_{3}\right)$ \\
\hline & Lime $(\mathrm{CaO})$ \\
\hline & Mix Srebrodolskite and $\mathrm{CaSi}$ \\
\hline & Mix Wue and $\mathrm{CaSi}$ \\
\hline
\end{tabular}

Figure 3: (a) Automated grouping of spectra. Red color shows a group of non-relevant spectra containing signals from both grains and epoxy mounting material. (b) Re-coloring phases and non-phase regions for better recognition. (c) Legends. Image width $=512 \mu \mathrm{m}$. number of maps that can be combined in one overlay image is limited to three, otherwise there is no unique correspondence between a given element combination and a given color. For complex materials with phases potentially comprising more than three elements (or oxide components), the visual overlay method is somewhat cumbersome and requires cross-referencing between different overlay permutations to identify all the phases present. A preferable option is to group pixels according to recurring combinations of multiple elements (chemically defined phases) and represent these in a color-coded segmented "phase map," while extracting the sum spectra of the grouped pixels to provide chemical information of the individual phases.

The phase identification approaches used in commercially available software can be divided into two main categories: grouping of areas in elemental X-ray maps [7] and principal component analysis (PCA) of the SI data cube $[8,9]$. In the first approach elemental maps are extracted after (or during) an SI data set acquisition. As soon as sufficient counts are acquired, phases can be defined and displayed for those spatial domains with similar element combinations. Individual spectra of a domain are summed to represent a phase spectrum. This selection can be done semi-automatically, by defining only the elements on which the grouping is to be based, or manually by selecting data domains in scatter plots or bivariate histogram plots of one element intensity/concentration against another. In addition, as spectra are quantified, ZAF maps can also be derived [10]. In the second approach a minimum number of principal spectral components are found, which are subsequently translated into maps to completely describe the SI data set. A detailed description of the PCA as used in the Noran System Six software (COMPASS from Thermo Fisher Scientific) is described in a technical note [9]. This approach has drawbacks, including for example that ZAF correction is not strictly applicable to spectra from principal component groupings because these do not necessarily represent physical entities (that is, phases) in a material. Additionally, the options for batch data evaluation of multiple image fields are limited because the principal components are derived for each individual image rather than being derived in a selected reference image and then applied to all subsequent images.

The approach used in PARC falls under the first category. Broadly speaking, the procedure is as follows: all individual pixel spectra are split into channels for evaluation, and when channel intensities exceed a userdefined threshold value, a peak position is defined and assigned to an element. Subsequently, spectra with 
Table 1: Phase comparison between PARC and XRD.

\begin{tabular}{|c|c|c|c|c|c|c|c|c|}
\hline & \multicolumn{2}{|c|}{ PARC } & \multirow{2}{*}{\multicolumn{2}{|c|}{$\begin{array}{c}\text { XRD-Rietveld } \\
\text { Bulk }\end{array}$}} & \multicolumn{2}{|c|}{ PARC } & \multirow{2}{*}{\multicolumn{2}{|c|}{$\begin{array}{c}\text { XRD-Rietveld } \\
\text { Bulk }\end{array}$}} \\
\hline & K1-Grain1 & K1-Grain2 & & & K3-Grain1 & K3-Grain2 & & \\
\hline & ave wt $\%$ & ave wt\% & $w t \%$ & $2 \sigma$ error & ave wt\% & ave wt\% & $w t \%$ & $2 \sigma$ error \\
\hline Lime (CaO) & 1 & 1 & 1.1 & 0.4 & 5 & 3 & 7.1 & 0.5 \\
\hline $\begin{array}{l}\text { Portlandite } \\
\left(\mathrm{Ca}[\mathrm{OH}]_{2}\right)\end{array}$ & & & 0.1 & 0.1 & & & 0.6 & 0.3 \\
\hline $\begin{array}{l}\text { Calcite } \\
\left(\mathrm{CaCO}_{3}\right)\end{array}$ & & & 0.1 & 0.2 & & & 0.5 & 0.4 \\
\hline $\begin{array}{l}\text { Aragonite } \\
\left(\mathrm{CaCO}_{3}\right)\end{array}$ & & & 0.0 & 0.3 & & & 0.7 & 0.4 \\
\hline Wuestite (FeO) & & & 6.5 & 2.0 & 31 & 26 & 18.6 & 1.8 \\
\hline $\begin{array}{l}\text { Mg-Wuestite } \\
\text { ([Mg,Fe]O) }\end{array}$ & 24 & 24 & 16.9 & 1.8 & & & 6.8 & 2.2 \\
\hline $\begin{array}{l}\text { Srebrodolskite } \\
\left(\mathrm{Ca}_{2} \mathrm{FeO}_{5}\right)\end{array}$ & 22 & 23 & 22.2 & 1.0 & 23 & 21 & 21.5 & 1.2 \\
\hline $\begin{array}{l}\text { Larnite } \\
\left(\mathrm{Ca}_{2} \mathrm{SiO}_{4}\right)\end{array}$ & 52 & 51 & 40.4 & 1.9 & 28 & 30 & 24.6 & 1.6 \\
\hline $\begin{array}{l}\mathrm{a}^{\prime}-\mathrm{C} 2 \mathrm{~S} \\
\left(\mathrm{Ca}_{2} \mathrm{SiO}_{4}\right)\end{array}$ & & & 9.8 & 1.9 & & & 3.8 & 1.5 \\
\hline $\begin{array}{l}\text { "Hatrurite" } \\
\left(\mathrm{Ca}_{3} \mathrm{SiO}_{5}\right)\end{array}$ & & & & & 9 & 16 & 0.0 & 0.5 \\
\hline amorphous & & & 3.0 & 6.1 & & & 15.8 & 4.2 \\
\hline
\end{tabular}

identical peak combinations are grouped together as phases. The group definitions set up for one image can be applied to another image acquired with the same analytical conditions, and batch processing of SI data sets comprising multiple image fields is integral to the functionality.

Validation approaches. The quantified chemistry of correctly assigned crystalline phases recognized with PARC should correspond to a known stoichiometry, requiring that a phase like $\mathrm{NaCl}$ is (a) free from crystallographically incompatible elements like $\mathrm{Si}, \mathrm{Al}$, or $\mathrm{O}$, and (b) its cationto-anion ratio is in agreement with the known chemical formula of $\mathrm{Na}: \mathrm{Cl}=1: 1$. This notion provides an important consistency check for the quality of the phase assignment with PARC. With sufficient total specimen surface area covered by multiple image fields dependent on local heterogeneity, the data sets should become representative of the bulk material. Such bulk data sets therefore also open the way to cross-validation checks of results with independent bulk analyses. Two additional "bulk" consistency checks that can be applied are related to XRD-Rietveld analysis and bulk chemical composition. First, the inventory of PARC-defined crystalline phases and their proportions should be consistent with those determined independently by Rietveld analysis of the powder XRD pattern (where agreement can reasonably be expected between (micro)chemically defined and crystallographically defined phases). In the second consistency check, the PARC output of chemical compositions and area proportions of phases, in combination with corresponding phase densities, allows the bulk chemical composition to be calculated and compared with independent bulk chemical analyses (XRF, inductively coupled plasma optical emission spectrometry). Therefore three potential validation "checks" for PARC apply: individual phase stoichiometry, consistency with XRD-Rietveld phase inventory and proportions, and agreement with bulk chemistry [6].

Sample selection. Two typical converter slag samples, K1 and $\mathrm{K} 3$, obtained in single batches of steelmaking were selected. Testing showed them to differ substantially in their leaching properties and volume expansion tendency. Typical aggregate properties of K1-type slags include low-volume expansion in EN1744-1 tests and noticeable vanadium leaching in the CEN/ TS 14405 test; whereas, K3 type slags exhibit much higher expansion but no vanadium leaching.

Specimen preparation for microscopy and data acquisition. Specimens were prepared by mounting the slag grains in Struers EpoFix, grinding, and polishing to a flat surface according to standard procedures. Light optical microscopy (LOM) was carried out using a Carl Zeiss AxioImager microscope, with integrated AxioVision software 4.9. Thereafter, the sample was coated with approximately $10 \mathrm{~nm}$ of carbon for SEM investigation. The SI data were acquired with a JEOL JSM-7001F SEM equipped with two $30 \mathrm{~mm}^{2}$ SDD detectors (Thermo Fisher Scientific) and a NORAN-System7 with NSS.3.3 software. The accelerating voltage was $15 \mathrm{kV}$, and the beam current in the focused probe was $6.2 \mathrm{nA}$. The step size was $1 \mu \mathrm{m}$, with an individual SI field comprising $512 \times 384$ pixels. The acquisition time per field was 60 minutes. 

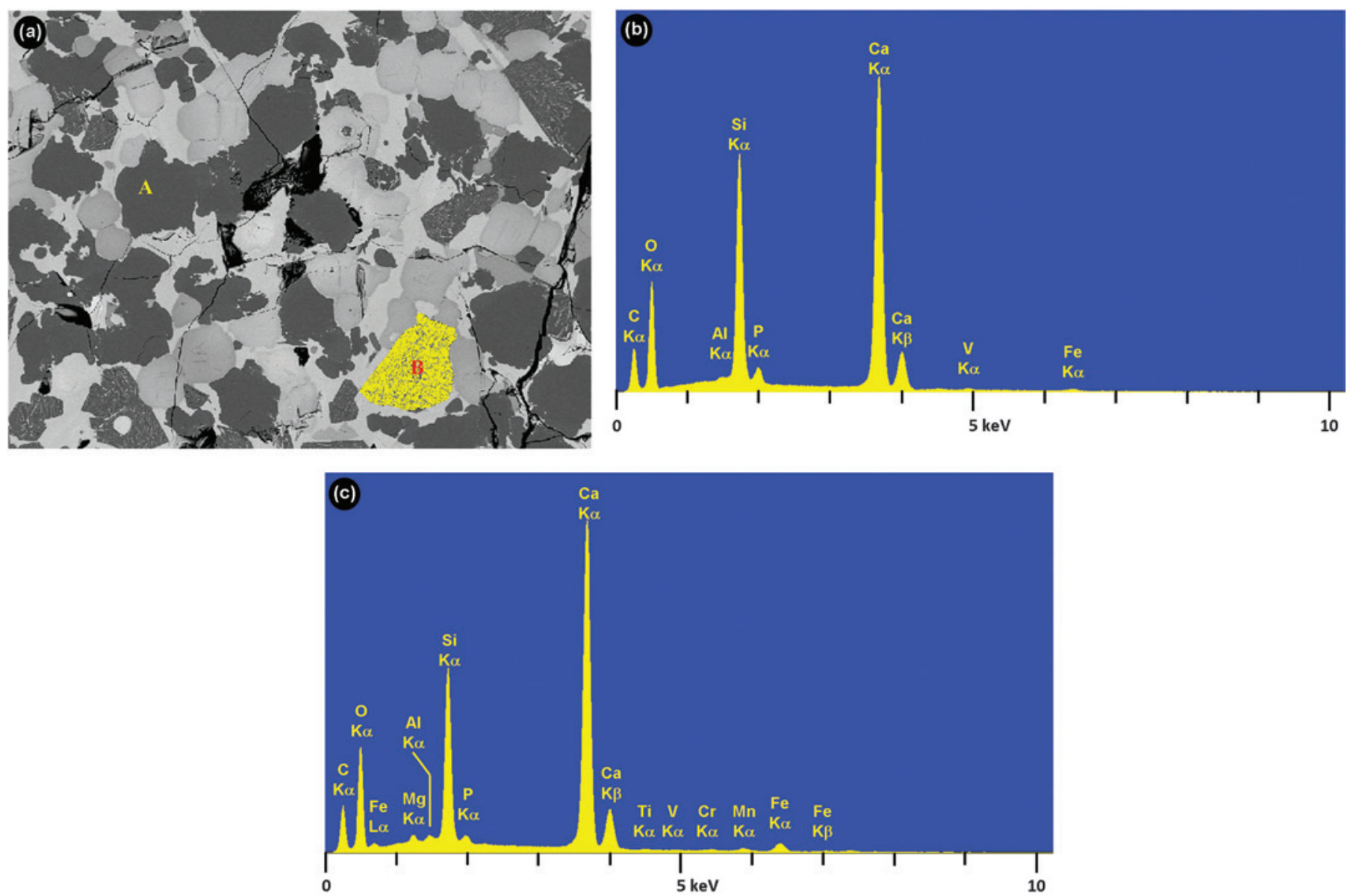

Figure 4: (a) Ca-silicate phases with different $\mathrm{Ca} / \mathrm{Si}$ ratio with representative grains A and B. Image width $=512 \mu \mathrm{m}$. (b) Sum spectrum extracted using NSS software from area A. (c) Sum spectrum extracted using NSS software from area B (yellow pixels).

\section{Results}

Preliminary LOM and SEM characterization. From each slag sample, a reflected-light LOM image was acquired (Figure 1) to select two representative grains for slag characterization. For each of these grains, SI data were acquired by SEM-EDS from more than 40 fields to cover the whole grain area. Figure 2 shows a backscattered electron (BSE) image of one of these fields from the K3 slag.

PARC finds and groups phases present. Prior to processing with the PARC software, SI data sets were converted into LISPIX format $[11,12]$. Next, a data set from a single SI image field was selected and imported into PARC for the initial phase characterization. For grouping spectra, the user-defined minimum energy cutoff was set to $0.9 \mathrm{keV}$, and a minimum signal threshold was selected. In the present example, the grouping of phases was based on the presence of (up to) five different X-ray peaks exceeding the threshold value (Figure 3a). Areas related to the epoxy mounting material or to non-relevant spectra from two phases at a phase boundary are coded in red. After initial grouping, a correlation table was produced (using the Pearson productmoment correlation coefficient), and groups with similar spectra were merged. For example, pixel spectra with a $\mathrm{K} \beta$ peak for $\mathrm{Ca}$ or Fe just below or just above a threshold value result in identification as different groups, and hence speckled domains in the phase images, while in fact they represent the same phase. These groups are automatically merged via the correlation step. For ease of visual phase recognition, color-coding of phases and non-phase regions may be listed in a legend according to the user's preferences (Figure 3b).

Separating similar phases with different element ratios. If within a group a subgroup is present with an identical combination of elements but in quantitatively different proportions, the group can be subdivided into multiple groups corresponding to the different phases. This is done by interactively selecting data regions in bivariate histogram plots-or "density plots" - of channel intensities or mathematical expressions thereof (for example, sum, ratio). Clustering of data in an $X-Y$ scatter plot is highlighted in an example here, for two different Ca-silicate phases/ phase-intergrowths (Figure 4). Phase A (grain A in Figure 2) shows an internally smooth appearance in the BSE image and exhibits the spectrum shown in Figure $4 \mathrm{~b}$. Phase B is in fact a two-phase intergrowth with a bright phase within a darker matrix. Extracting a clean phase spectrum of the darker phase within B via manual pixel selection using NSS software (Figure 4c) is cumbersome because there are always contributions of grain-edge pixels of the intergrown brighter phase and because 
the microstructure is finer than the analytical spatial resolution for X-ray spectrometry. It is actually more useful to identify the phase intergrowth (B) as the decomposition product of a former single phase, hence lumping together both the brighter and darker phases. This is possible in PARC: the broader group defined, by $\mathrm{Ca}, \mathrm{Si}$, and $\mathrm{O}$ peaks, can be subdivided into two phases using the density plot approach. As demonstrated in Figure $5 \mathrm{a}$, a polygon selection of a Ca-rich phase is defined in such a way that this phase corresponds to those regions visible in orange on the BSE grayscale image (Figure $5 b$ ). In this way, distinction is made between phase $\mathrm{A}$, "C2S" $\left(\mathrm{Ca}_{2} \mathrm{SiO}_{4}\right)$, and phase $\mathrm{B}$, "C3S" $\left(\mathrm{Ca}_{3} \mathrm{SiO}_{5}\right)$. The latter exhibits an exsolution intergrowth of $\mathrm{C} 2 \mathrm{~S}$ (dark phase) and lime ( $\mathrm{CaO}$, bright phase), a phase mixture commonly encountered in such slag material. Once all groups are refined and the desired color-coding legend is specified, this model can be stored and applied to all SI data sets collected under identical analytical conditions. An example of the final color-coded phase segmentation map of a single K3 grain is shown in Figure 6.

Pixel erosion at boundaries. All pixels are included for the purposes of producing phase maps out of image fields and for quantifying areal proportions (volume fractions) of phases. However, pixels adjacent to other phases may contain a mixed chemical signal reflecting both phases. For the purposes of extracting "clean" spectra for quantitative chemical analysis of the individual phases, these pixels must be excluded. This is achieved by applying a binary erosion step using a $3 \times 3$ matrix. Only those pixels whose eight neighboring pixels are all allocated to the same group contribute to the sum spectrum. In sample K3, if the C3S phase intergrowth (phase B) had been further subdivided into its constituent two separate phases, this erosion step could be used to extract cleaned-up spectra of the two (if any pixels remained after erosion). In the case of phase $\mathrm{B}$ in Figure 4, the bright $\mathrm{CaO}$ regions each occupy only a few pixels and may be smaller than the analytical spatial resolution at $15 \mathrm{kV}$.

Identification of boundary phases. In the $\mathrm{K} 1$ slag sample, $\mathrm{V}$-rich entities are expected. To extract these from the C2S phase, a density plot was generated using the Si signal on the $X$ axis and the sum of $\mathrm{V}+\mathrm{P}+\mathrm{Al}$ signals on the $Y$ axis (Figure 7a). A polygon was drawn manually around the pixels with high intensities of $\mathrm{V}+\mathrm{P}+\mathrm{Al}$ and defined as a separate group (colored pixels in Figure 7b). Many of these green pixels (354 in total) were immediately adjacent and contaminated by the signal from an Al-rich Ca-ferrite phase, inferred from their relatively high Al-signal. After applying an erosion filter to the pixels within the red square, only seven pixels remain, coded in red in Figure 7c, from which a cleaned spectrum of the V-rich sub-group could be extracted. A "before and after" comparison between the two extracted spectra (Figures 7d) shows clearly the effect of the erosion filter in cleaning up the phase spectrum extraction. The combined approach of using density plots and the erosion filter is extremely useful for removing (even minimal) contamination of analyses from adjoining phases.

Large-area phase images. Once a model has been established, it can be run in batches on series of SI fields collected under the same conditions that can subsequently be stitched together to

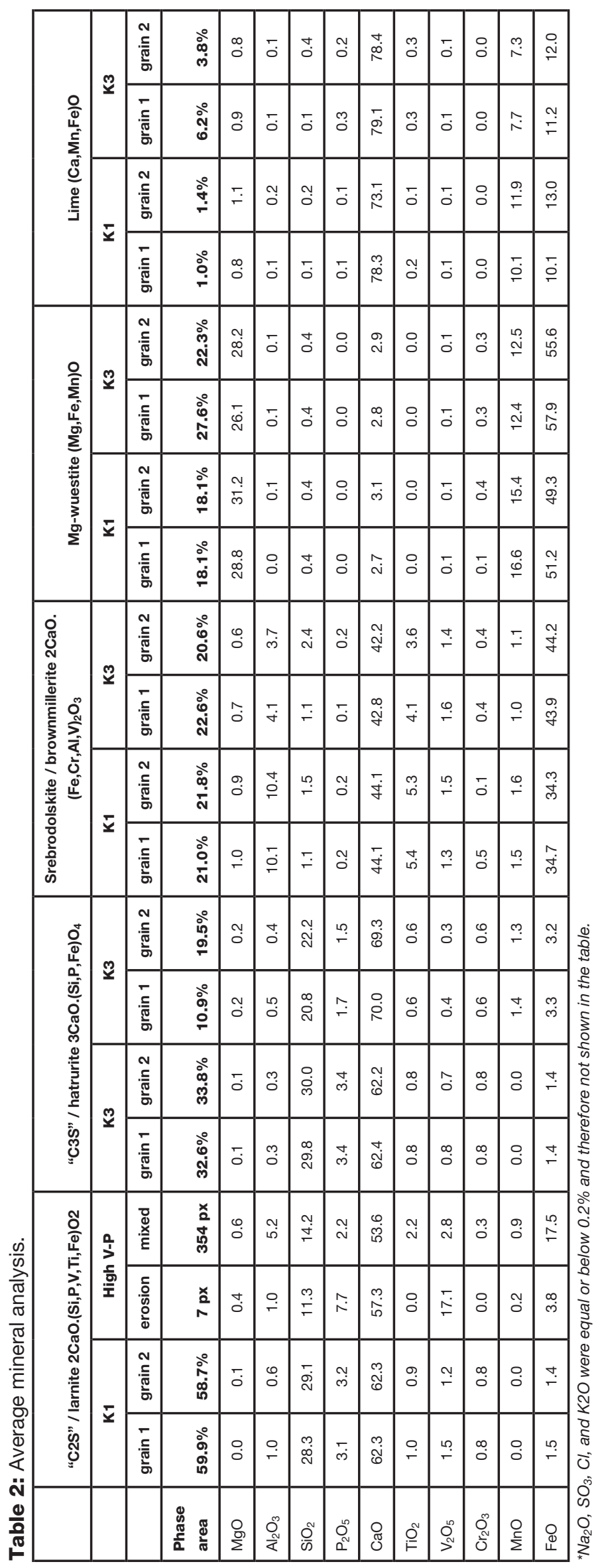




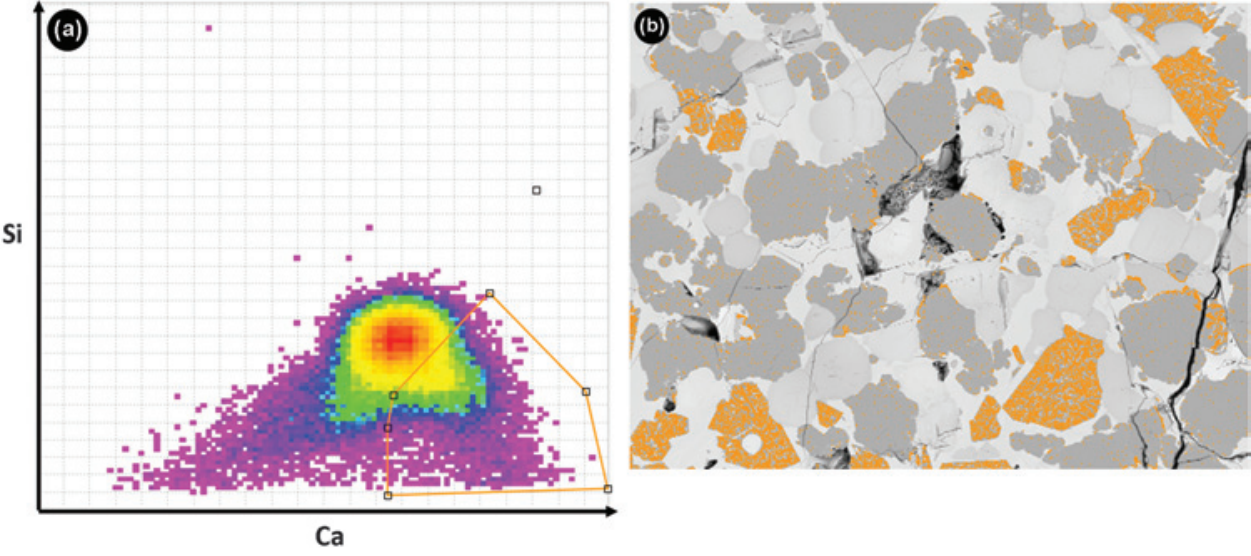

Figure 5: (a) Density plot of $\mathrm{Si}$ and $\mathrm{Ca}$ for Ca-rich group of pixels only. Polygon selection of pixels tuned to correspond with BSE image in Figure 5b. (b) BSE image with selected pixels from density plot in highlighted orange. Image width $=512 \mu \mathrm{m}$.

create phase segmentation images several millimeters across (Figure 8). This particular example shows clearly the length scale of heterogeneity of slag grains for $\mathrm{K} 1$ and $\mathrm{K} 3$. The areal proportions of each individual phase can be exported per SI field. When integrating over sufficient surface area (if necessary multiple SI fields), estimates of local bulk composition, and any systematic gradients therein, may be obtained. In this case, no gradients in phase distribution were observed on the length scale of an individual grain, and the areal proportions of phases were integrated over each grain as an indication of the slag's bulk mineralogy (Table 1). The phase proportions (Table 1), phase chemistries from the extracted phase spectra (Table 2), and assumed phase densities are combined to calculate the bulk chemical composition of each grain (Table 3). The total time to characterize a slag grain, 45 fields as presented in Figure 8d, including SI data acquisition (1 hr per field) and modeling with PARC (for this application $\sim 5$ minutes), was between 46 and 47 hours depending on the computer platform (MAC or PC) used.

\section{Discussion}

Validation. Qualitatively, the same major phase categories are identified by both PARC and independent XRD analyses the major elements $(\mathrm{Si}, \mathrm{Ca}, \mathrm{Mg}$, and $\mathrm{Fe}$ ), whereas for minor elements this deviation ranges up to $20 \%$ relative (for example, $\mathrm{Ti}$ ).

The quantitative discrepancy between PARC and XRD phase proportions (Table 1) in the case of the Ca-silicates, Ca-(hydrous)oxide, Ca-carbonate, and $\mathrm{Mg}$-Fe-oxides is explained by the chemical versus crystallographic definition of phases in the respective analytical methods, which in fact highlights the necessity of combining independent techniques to fill the blind spots in each. The phase intergrowth referred to in previous sections is defined in PARC as a single phase-C3S - while in fact the intergrowth represents a well-documented decomposition texture of $\mathrm{C} 3 \mathrm{~S}$ to a mixture of $\mathrm{C} 2 \mathrm{~S}$ and lime $(\mathrm{CaO})$, apparently amorphous because it does not show up as additional $\mathrm{C} 2 \mathrm{~S}$ and lime, but corresponds to the "amorphous" content in the Rietveld XRD analysis. The absence of any detectable crystalline C3S phase (hatrurite) in the XRD analyses is therefore explainable. The higher proportion of lime, portlandite, and Ca-carbonate in sample $\mathrm{K} 3$ corresponds well with the higher fraction of " $\mathrm{CaO}$ " in the PARC analysis (all three phases in the XRD would be identified as $\mathrm{CaO}$ using the PARC chemical definition of $\mathrm{Ca}$ and $\mathrm{O}$

Table 3: Bulk chemistry comparison between PARC and XRF.

\begin{tabular}{|c|c|c|c|c|c|c|}
\hline & \multicolumn{2}{|c|}{ PARC } & $\frac{\text { XRF }}{\text { K1-Bulk }}$ & \multicolumn{2}{|c|}{ PARC } & $\begin{array}{c}\text { XRF } \\
\text { K3-Bulk }\end{array}$ \\
\hline & $w t \%$ & $W t \%$ & $w t \%$ & $W t \%$ & $W t \%$ & $W t \%$ \\
\hline $\mathrm{Al}_{2} \mathrm{O}_{3}$ & 2.8 & 2.7 & 2.7 & 1.1 & 1.0 & 1.1 \\
\hline $\mathrm{SiO}_{2}$ & 15.2 & 15.4 & 14.8 & 10.4 & 13.0 & 11.9 \\
\hline $\mathrm{CaO}$ & 43.7 & 43.8 & 44.7 & 38.2 & 41.8 & 43.6 \\
\hline $\mathrm{TiO}_{2}$ & 1.7 & 1.7 & 1.4 & 1.2 & 1.1 & 1.0 \\
\hline $\mathrm{V}_{2} \mathrm{O}_{5}$ & 1.1 & 1.0 & 1.2 & 0.6 & 0.6 & 0.8 \\
\hline $\mathrm{Cr}_{2} \mathrm{O}_{3}$ & 0.6 & 0.5 & 0.2 & 0.5 & 0.5 & 0.2 \\
\hline $\mathrm{MnO}$ & 4.6 & 4.3 & 4.7 & 5.2 & 4.4 & 4.5 \\
\hline
\end{tabular}

${ }^{*} \mathrm{Na}_{2} \mathrm{O}, \mathrm{SO}_{3}, \mathrm{Cl}$ and $\mathrm{K}_{2} \mathrm{O}$ were equal or below $0.2 \%$ and therefore not shown in the table. 


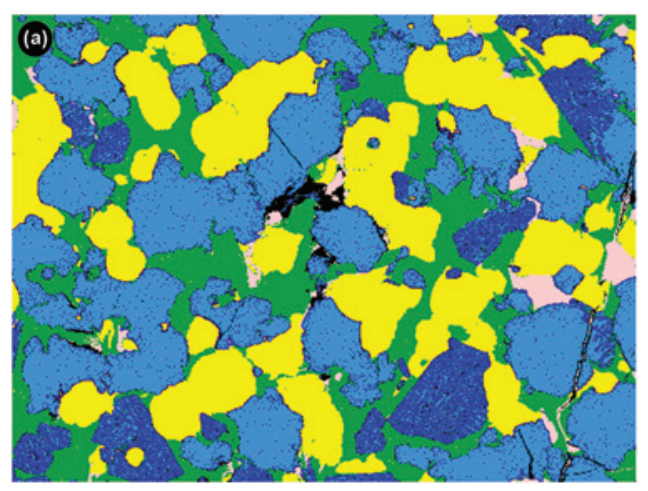

\begin{tabular}{|c|c|}
\hline (b) & Unclassified \\
\hline & Empty Spectra \\
\hline & Embedding \\
\hline & $\mathrm{C} 2 \mathrm{~S}\left(2 \mathrm{CaO} . \mathrm{SiO}_{2}\right)$ \\
\hline & $\mathrm{C} 3 \mathrm{~S}\left(3 \mathrm{CaO} . \mathrm{SiO}_{2}\right)$ \\
\hline & Mg-Wue $(\mathrm{Mg}, \mathrm{Fe}) \mathrm{O}$ \\
\hline & Srebrodolskite $\left(2 \mathrm{CaO} . \mathrm{Fe}_{2} \mathrm{O}_{3}\right)$ \\
\hline & Lime $(\mathrm{CaO})$ \\
\hline & Mix \\
\hline
\end{tabular}

Figure 6: (a). Final color-coding K3-grain1. Image width $=512 \mu \mathrm{m}$. (b) Legends.

peak combination). In short, this serves to illustrate that the PARC results can be cross-validated with other independent techniques and constraints and that in this case any discrepancies have a logical explanation given prior knowledge of the materials and associated processes.

Strategies for effective use of PARC. The results above illustrate how the PARC software can be used to identify phases and produce a large-area phase map. Often it is necessary to check for phases that are unexpected or to detect known phases that are present in low-volume fractions. These determinations can be made using PARC to process SI data sets via two different strategies: full-automatic mode and query mode.

Full-automatic mode. In full-automatic mode PARC sorts the individual pixel spectra according to their characteristic multi-element combinations using only two constraints: peak threshold above background and the X-ray energy lower limit cut-off. Generally this is a successful strategy with coarse-grained materials having phase domains of $10 \mu \mathrm{m}$ or larger, such as geological samples [1] and metallurgical slag. In full-automatic PARC mode, pixels within phases forming solid solutions with a broad chemical range can become assigned to separate phases. However these can be merged manually afterwards to a single phase if stringent phase mapping is desired. In general, a solid solution phase can be recognized independently by BSE-intensity changes within a grain. These BSE-intensity changes correspond
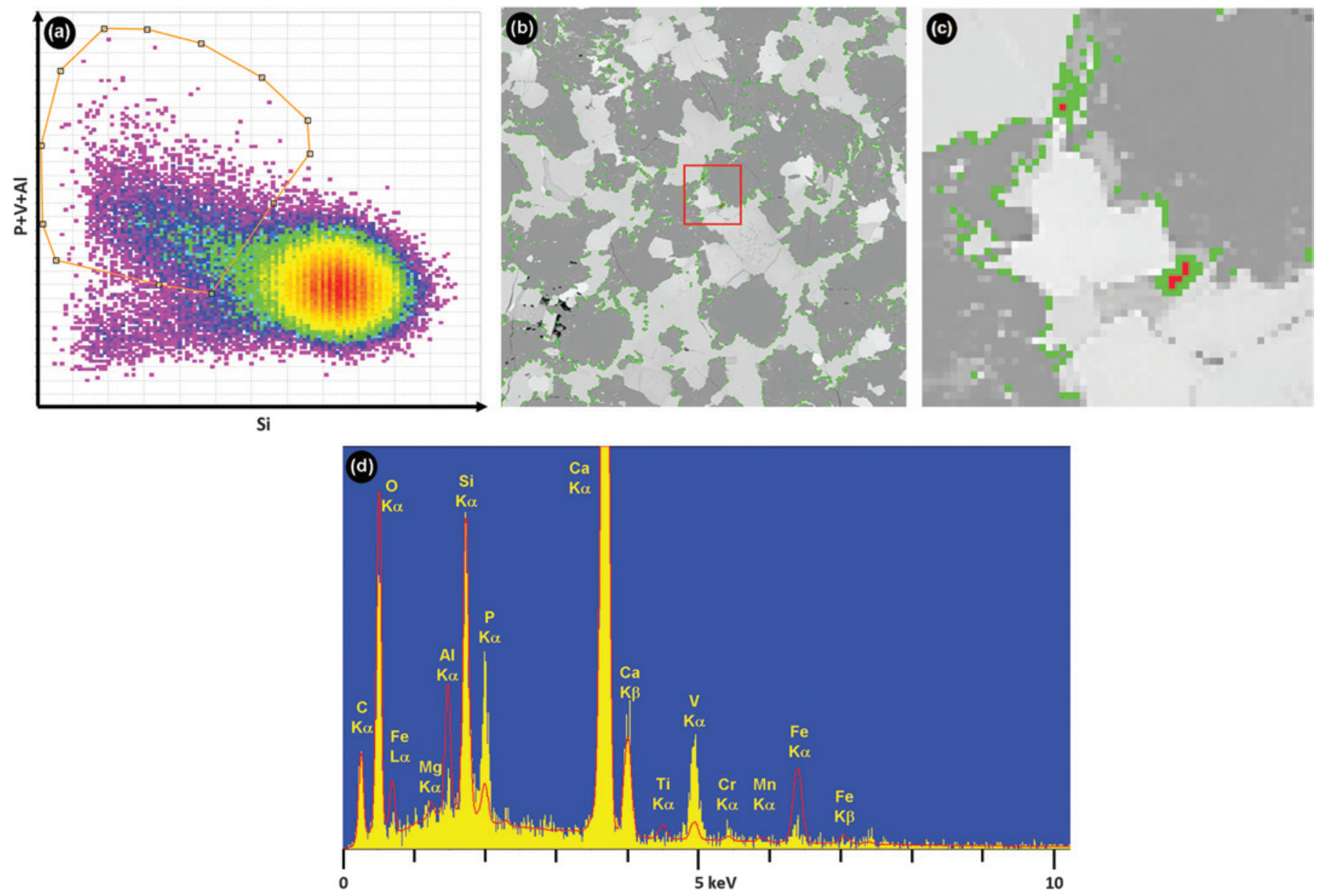

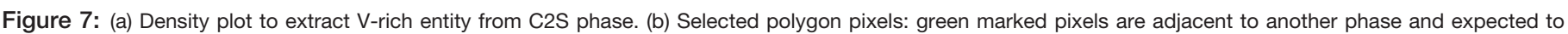

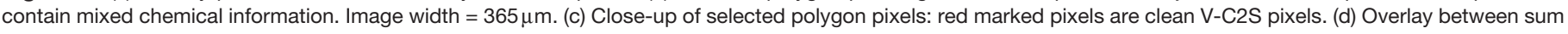
spectra of all selected polygon pixels (red spectrum) and clean pixels (yellow spectrum). Spectra are scaled to Ca-K $\alpha$. 

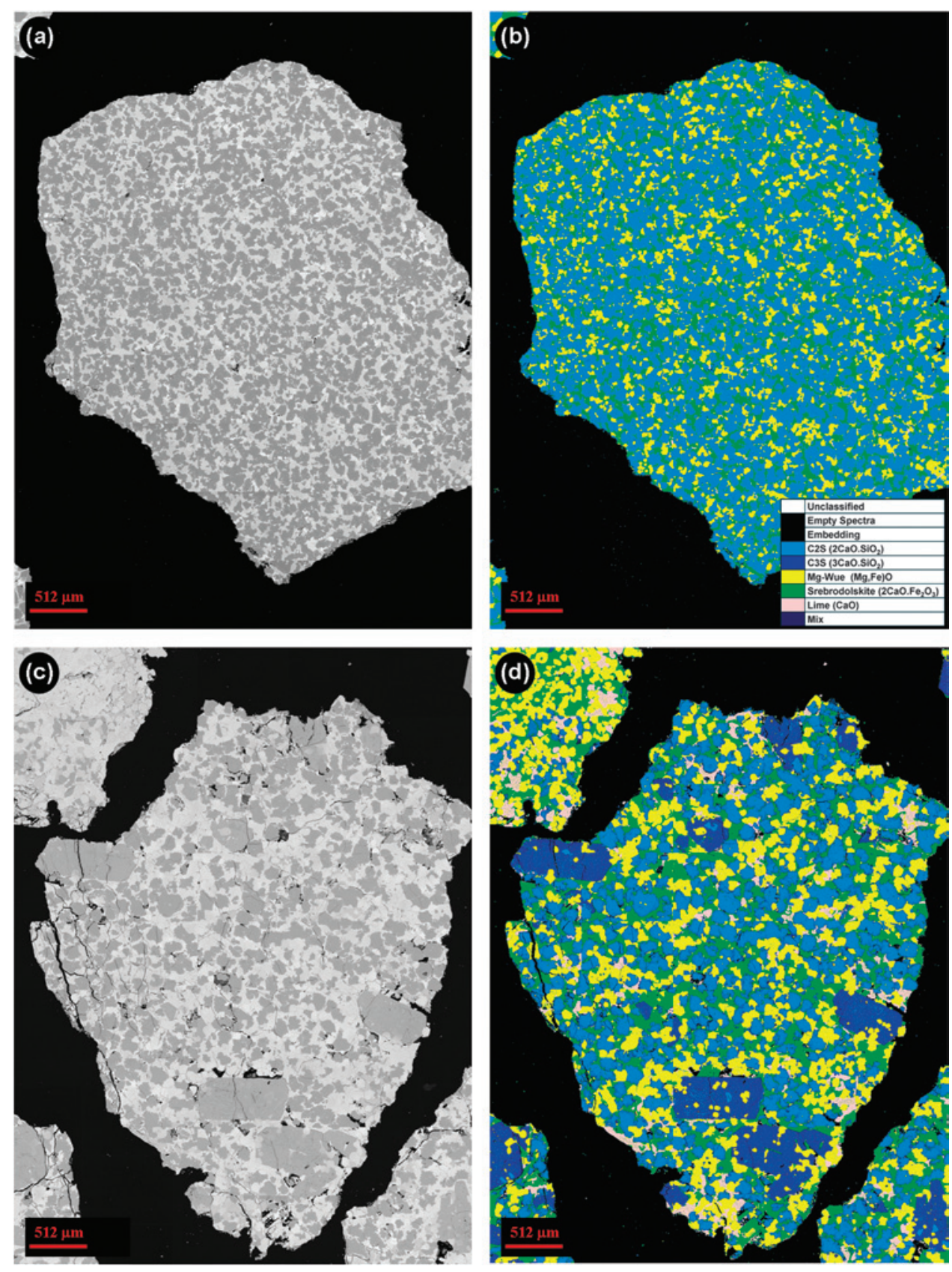

Figure 8: Large-area phase images. (a) BSE image of K1-slag grain. (b) Phase image of K1-slag grain including a legend. (c) BSE image of K3-slag grain. (d) Phase image of K3-slag grain (legend in (b) applies). Image width $=2.56 \mathrm{~mm}$.

to a variation of elements concomitant with a fixed stoichiometry linked to the phase's crystal structure. In PARC, compositional gradients in such solid solution phases can be highlighted visually in the phase segmentation maps with a color shading for a single element (even for those elements with an energy below the minimum defined $\mathrm{keV}$ used for initial grouping), element ratio, or combination of elements. The full-automatic mode recognizes trace phases containing unusual elements or combinations of elements. Artifacts associated with adjoining phase boundaries can be mitigated by applying image-processing techniques such as erosion operations on the peripheries of phases.
Query mode. In the query mode, PARC is asked to search for known element combinations that are specific for a given phase of interest. This works well with prior knowledge about a sample (for example, from powder XRD-analysis), but it can also be used when searching for the presence of certain trace phases in large sample areas.

Typically both of these strategies would be employed: (1) use the software in automated mode to confront the user with unexpected features that may otherwise be overlooked, and (2) use the software in query mode to find expected features that may be so low in abundance as to be below the detection limits of other techniques (for example, bulk XRD-Rietveld). A key strength of PARC software is the flexibility for the user to readily modify and refine the phase assignment model manually using "density plots" as described.

The aim of developing PARC was to enhance our ability to harness SI data sets for characterizing the phase distribution and phase chemistry in diverse materials with respect to what was possible with commercially available software. The key functional features of PARC are summarized as follows: (a) automatic and query grouping of individual SI pixel spectra as phases, (b) flexibility in user-defined refinement of phase allocation by merging and/or splitting phases, (c) clean-up functionality (erosion) to obtain accurate phase chemistry, and (d) batch processing of multiple SI data sets with a given phase allocation model.

The introduction of PARC software at Tata Steel R\&D has fundamentally changed our approach to materials characterization. Our PARC phase assignment models have been developed for various types of steel industry-related materials, such as iron-ore pellets [13], metallurgical slags [14-16], diverse process and environmental dusts, casting powder, and surface defects in steel products. In addition, PARC has been applied to geological investigations (experimental petrology [2, 17] on hydrous andesite and gabbro), archeological and historical artifacts (Roman steel, seventeeth-century paintings [1]), and slags from several other processes, such as sewage-sludge incineration and fertilizer production. 


\section{Conclusion}

Developments in SEM and SI have made it possible to acquire micro-spatially resolved chemical information on solid materials so quickly and in such large volume that a major challenge is how to process the data to unlock the stored information. An innovative software approach has been described here for automating phase recognition, producing phase segmentation maps, quantifying areal phase proportions, and extracting phase spectra from large SI data cubes: PARC. Key to the functionality of the software is the combination of (1) automated phase recognition based on elemental combinations in pixel spectra, (2) flexibility for user-defined refinement of phase allocation models and clean-up of extracted phase spectra, and (3) batch processing on multiple SI fields in an unattended mode. Complex microstructures can be clearly visualized, and phase proportions and phase chemistry accurately quantified, on a range of length scales (up to several millimeters). This enables a bridge to be made between microanalysis and bulk analyses: heterogeneities and gradients in local bulk mineralogy and chemical composition can be quantified that would otherwise fall between the two length scales of analysis. The PARC approach moreover lends itself to crossvalidation with bulk techniques (for example, bulk XRF, XRD analyses).

\section{References}

[1] C van Hoek et al., Microsc Microanal 17 (2011) 656-60.

[2] C van Hoek et al., European Microbeam Analysis Society (2013) p. 40.
[3] C van Hoek et al., Mineralogical Magazine 75(3) (2012) 2068.

[4] EUROSLAG, “REACH,” http://www.euroslag.com/ status-of-slag/reach.

[5] SR van der Laan et al., 2nd International Conference on Accelerated Carbonation for Environmental and Materials Engineering, Rome, Italy, p. 229-38.

[6] SR van der Laan et al., Metall Mater Trans B (in preparation).

[7] JJ Friel and CE Lyman, Microsc Microanal 12 (2006) 2-25.

[8] PG Kotula et al., Microsc Microanal 9 (2003) 1-17.

[9] P Camus, Thermo Fisher Scientific, Technical Note White paper 51782.

[10] R Wuhrer et al., European Microbeam Analysis Society, workshop, Porto, Portugal, 2013.

[11] DS Bright, Lispix, a PC-based image processing engine, http://www.nist.gov/lispix.

[12] DE Newbury and DS Bright, SCANNING 27 (2005) 15-22.

[13] C van Hoek, Microsc Microanal 20(suppl. S3) (2014) 660-61.

[14] E Berryman et al., Mineral Mag 76(6) (2012) 1479.

[15] J Riker et al., Mineral Mag 75(3) (2012) 1725.

[16] A van Zomeren et al., Waste Manag 31(11) (2011) 2236-44.

[17] SR van der Laan et al., Mineral Mag 75(3) (2012) 2063.

\section{How to Identify and solve Your SIMI Contamination Problem}

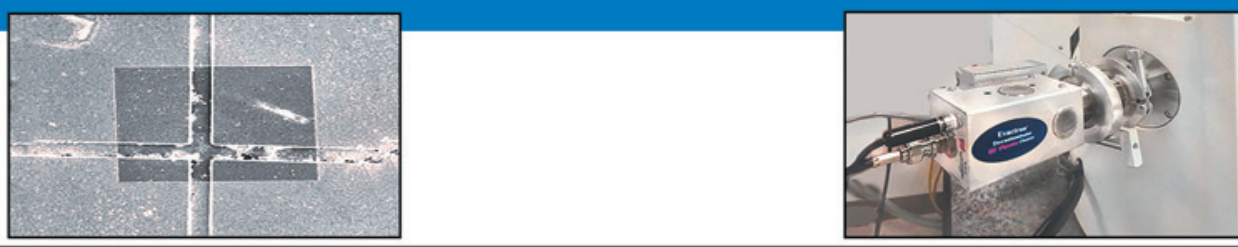

\section{Contamination Artifact: Black Square $=$ Dirty Chamber}

Evactron ${ }^{\circledR}$ Solution: EP Remote Plasma Cleaner

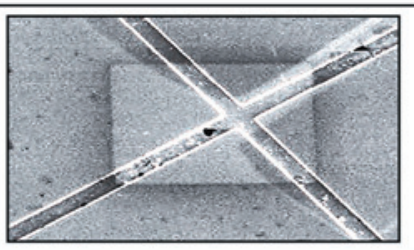

Contamination Artifact: Dark Border $=$ Dirty Sample
Evactron ${ }^{\circledR}$ Solution: Easy Plasma Sample Cleaner (EPSC)

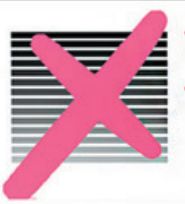

Find your Fastest Way to Pristine at EVACTRON.COM 1-650-369-0133 


\section{lumencor}

light engines for a

BRIGHTER. GREENER. PLANET.

\section{BRINGING NNOVATION}
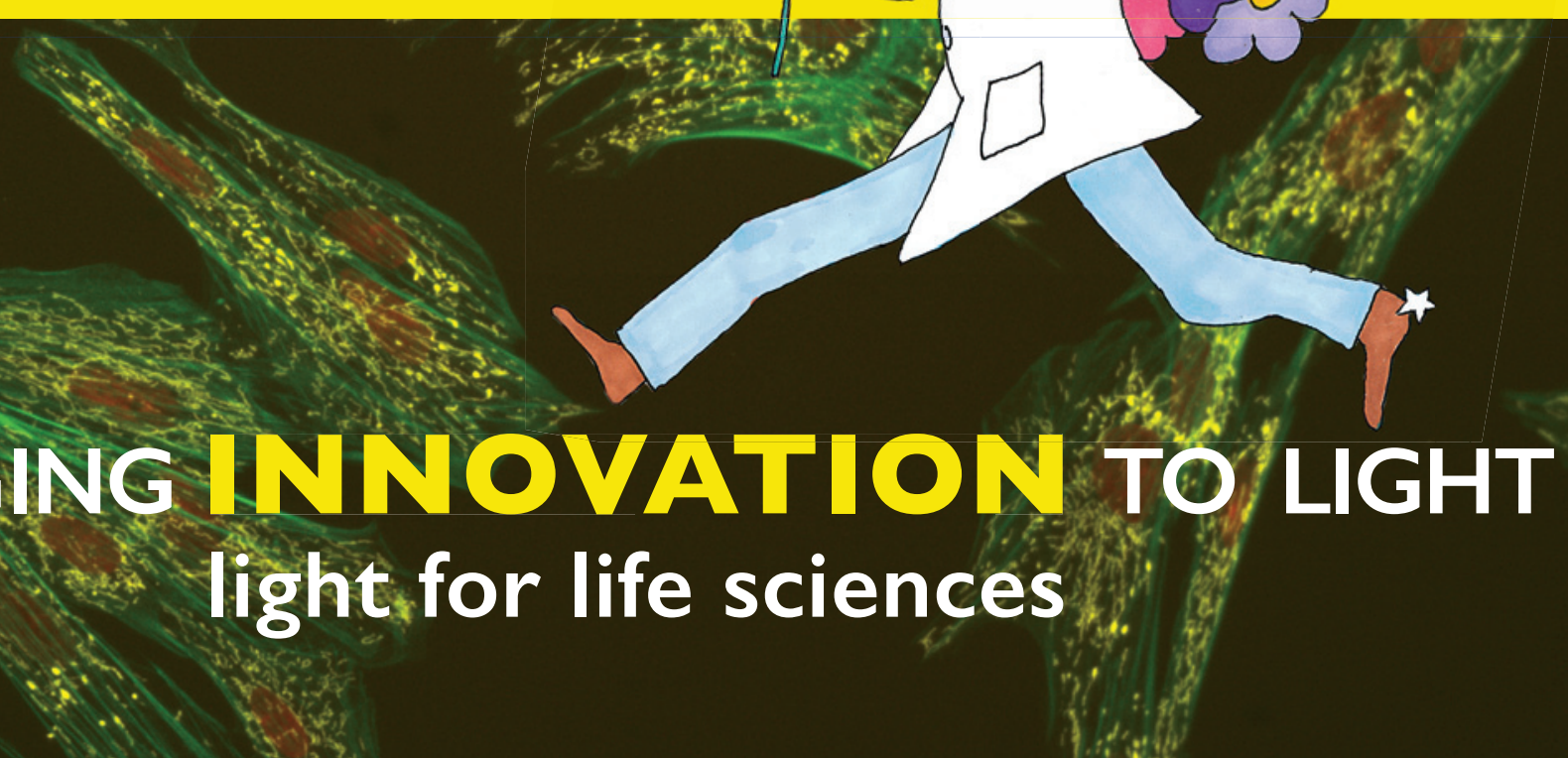

SPECTRA $X$ light engine ${ }^{\circledR}$ Power, Speed and Flexibility

- Integrated array of six powerful solid-state light sources

- User exchangeable filters to refine spectral output

- Fast hardware triggering up to $5 \mathrm{kHz}$

- Software controlled color output, intensity and timing

- Liquid light guide or optical fiber output coupling

- Optional nIR, quantitation and hand held control POD

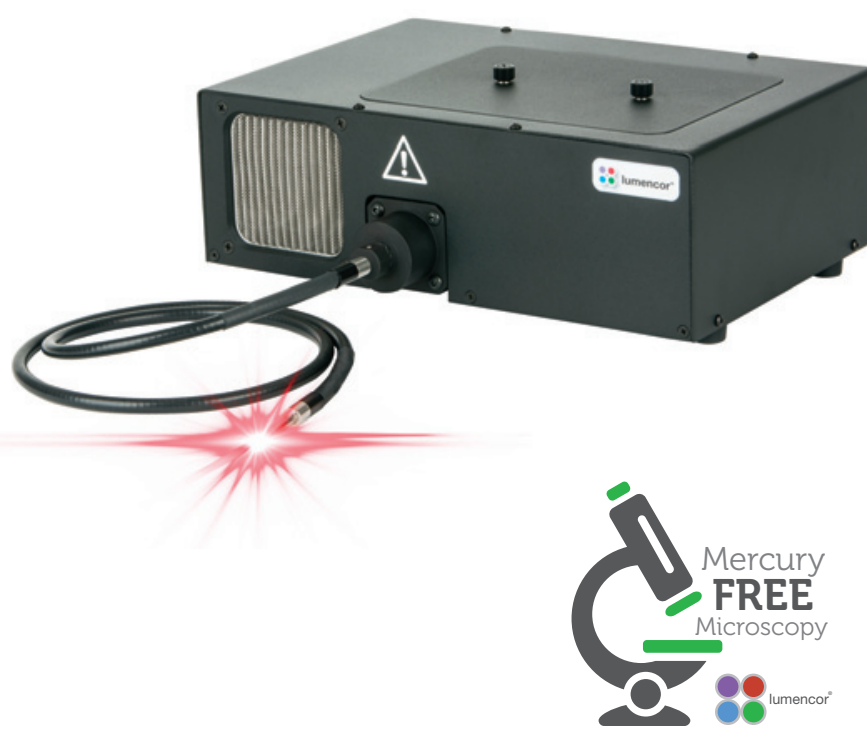

Interested in learning more?

Find a full list of features, applications and specifications on our website. 